\title{
Validation of UGV autonomous navigation based on an adapted path planning algorithm using UAV imagery.
}

\author{
Francesco Messina1, Federico Faedda1, Vincenzo Di Pietra ${ }^{1,2}$, Andrea Lingua ${ }^{1,2}$ \\ ${ }^{1}$ PIC4SeR-Politecnico di Torino Interdepartmental Centre for Service Robotics, Torino, 10129, \\ Italy \\ ${ }^{2}$ Department of Environment Land and Infrastructure Engineering (DIATI), Politecnico di Torino, \\ Torino, 10129, Italy
}

Correspondence to: Vincenzo Di Pietra ${ }^{1,2}$ (vincenzo.dipietra@polito.it)

https://doi.org/10.31490/9788024845050-4

\begin{abstract}
Several fields benefits from the use of automated machines and in particular from the advances in autonomous navigation. One of these is agriculture, which has been using Global Navigation Satellite System (GNSS) positioning systems and navigation algorithms in land machinery for years. Path planning is considered a fundamental step for autonomous agricultural machines, especially in the vineyard, where the terrain morphology and obstacles represent a big challenge. This paper proposes a new method of using the path planning algorithm Rapidly-exploring Random Tree* $\left(R T^{*}\right)$ as the core of a more complex superstructure and validate its usability in a real scenario. Firstly, a commercial Radiocontrolled $(\mathrm{RC})$ vehicle has been modified, implementing the required hardware components and sensors for autonomous driving. Secondly, an orthomosaic and a digital terrain model (DTM) obtained by an Uncrewed Aerial Vehicle (UAV) photogrammetric survey are used to identify the vine rows and to store information about the slope variation. The code makes use of these data in order to generate waypoints used later by the autopilot to navigate. The last step is the prototype testing and the validation of the navigation accuracy.
\end{abstract}

\section{INTRODUCTION}

In the agricultural sector, to increase productivity and safety in procedures related to moving vehicles, new technological systems have recently been implemented that relies on wellknown navigation technologies such as GNSS and its hybridization (Wang, H., \& Noguchi, $N$.). Other active and passive sensors have been used to increase the context-awareness of these vehicles (Zhang, Z., et al.), while information systems based on spatial information are used for planning and analysis procedures (Emmi, L., et al.). In this context, path planning algorithms play a central role in the architecture used for the realization of autonomous vehicles (Michael W Otte.).

In this work, a commercial RC vehicle was used to autonomously navigate the uneven terrain of a vineyard, integrating low-cost sensors on-board. The navigation board operates offline following the path obtained by the proposed code which exploits the RRT* path planner (Karaman, S., \& Frazzoli, E.). The latter manages to accomplish its task also thanks to the collaboration with a UAV that, utilizing the aerial photogrammetric survey method, can 
generate high-precision georeferenced orthophotos that are later used by the proposed code. A mission was planned in a real scenario to test the results of the path planning and to validate the navigation accuracy.

\section{MATERIALS AND METHODS}

The vehicle selected is the Traxxas X-Maxx, a very compact $(779 \times 540 \times 350 \mathrm{~mm}$ and $10 \mathrm{~kg}$ in weight) $\mathrm{RC}$ vehicle, which ensures particularly good rollover stability and allows for maneuvering even in small spaces. Navigation is delegated to the ArduPilot Pixhawk 1 navigation board which has a large number of I / $\mathrm{O}$ interfaces suitable for connecting numerous sensors. In the proposed system, the Piksi Multi dual-frequency multiconstellation GNSS receiver is the main positioning and navigation system, while a secondary ublox receiver was installed for redundancy reasons (Fig. 1).
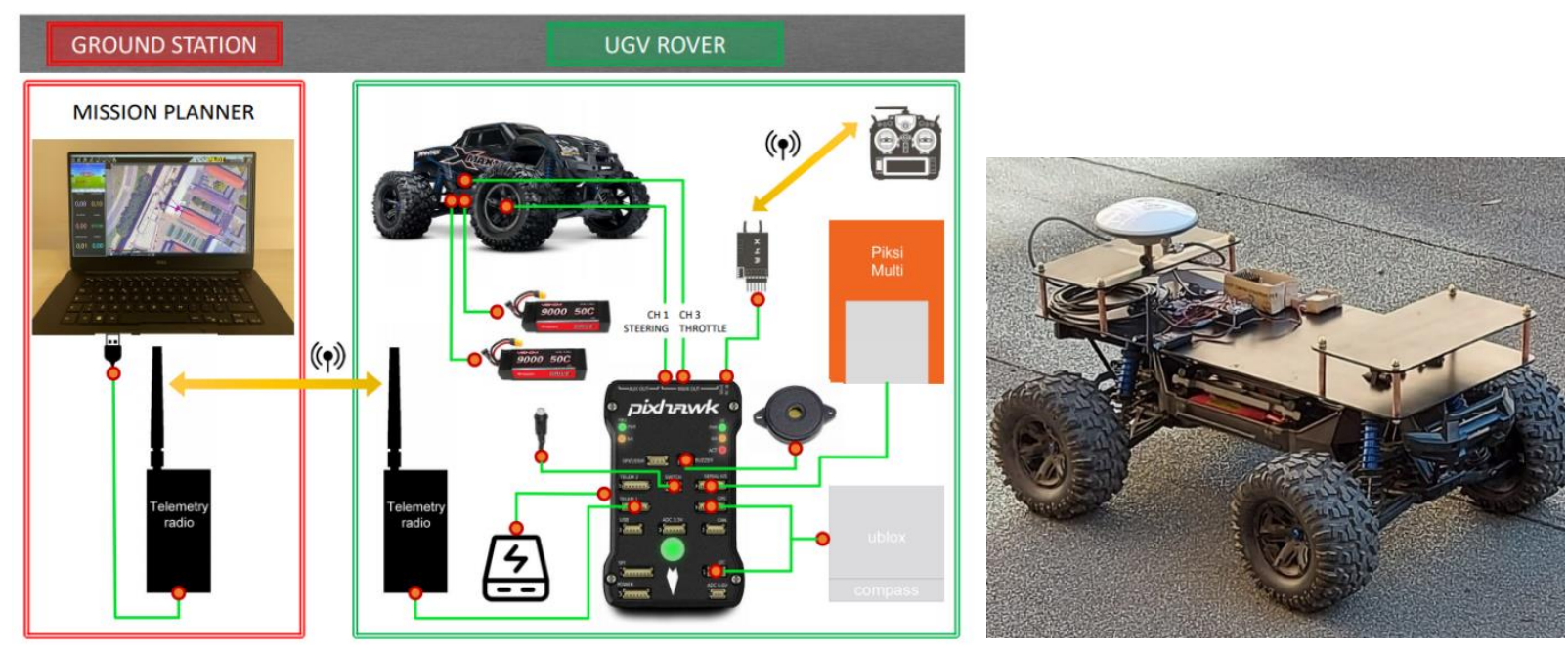

Fig. 1. (Left) UGV customization, with communication scheme, sensors and ground control station and its realization (right).

\section{Path Planning Algorithm}

The $\mathrm{RRT}^{*}$ algorithm samples random points in the configuration space and connects them incrementally building tree branches and it is biased to grow towards large unsearched areas of the graph. The planning search space is represented through a costmap, a binary map that contains information about the surroundings, possible obstacles as well as areas that cannot be traversed by the vehicle represented by an inflation radius created around obstacles. The costmap is stored as $2 \mathrm{D}$ grid of cells, which has values ranging from 0 to 1 representing the cost of navigation through that grid cell. Around the $\mathrm{RRT}^{*}$, a set of algorithms has been created to minimize the distance and at the same time evaluating how effective the path is, in terms of slope, slope variation and roll in a trial and error fashion. To create the costmap and to perform the validity check apriori information of the environments are required. Therefore, through an aerial photogrammetric survey from an UAV, georeferenced and metrically accurate information maps of an agricultural site were produced. The orthomosaic and the DTM obtained are subsequently used to identify the rows of a vineyard (Zoto, J., et al.), to extract high resolution elevation information and to georeferencing the costmap (Fig. 2). The code dynamically explores most of the free 
configuration space to find a route through which the vehicle will reach the final position from the starting one.
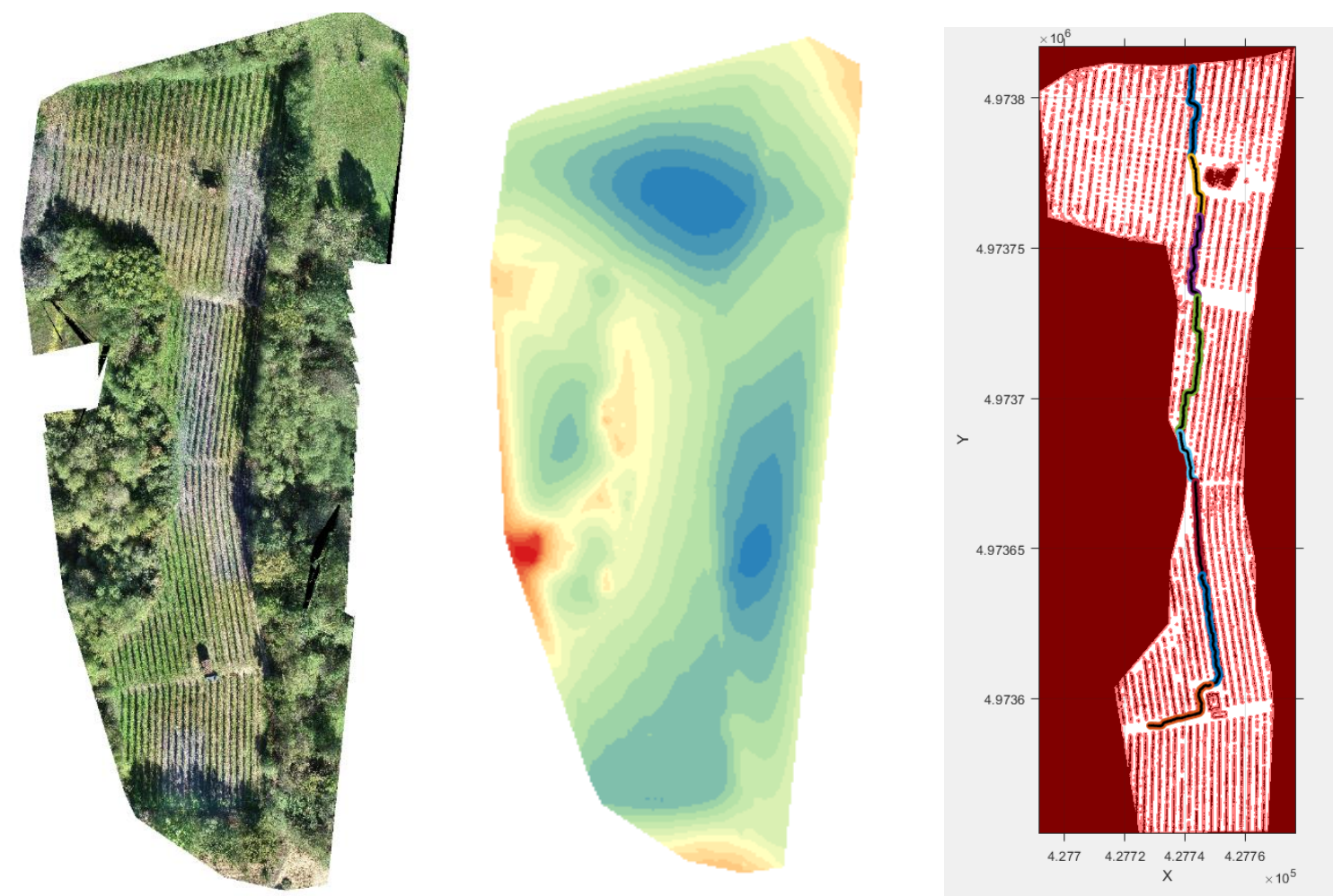

Fig. 2. (Left) An orthomosaic of a vineyard and (Center) a DSM obtained by the UAV survey. (Right) The georeferenced costmap with an example of planned path.

The path thus found is analysed pose by pose referring to the real point of contact between the vehicle surface and the ground, i.e. the wheels, ideally overlapping the 2D binary mask with the DTM that contains elevation data (Fig. 3).

By doing so, the code can examine whether to discard the newly found route using the constraints entered by the user as evaluation criteria. This method of exploration is perfectly suited to sample-based algorithms, in which an implicit method of environment representation is used, i.e. determining whether a point in the configuration space is valid, using a black-box function. The last code section is devoted to the creation of the waypoints text file in MAVLink format which respects the imposed constraint and which allows the navigation board to perform a task using the GNSS information.
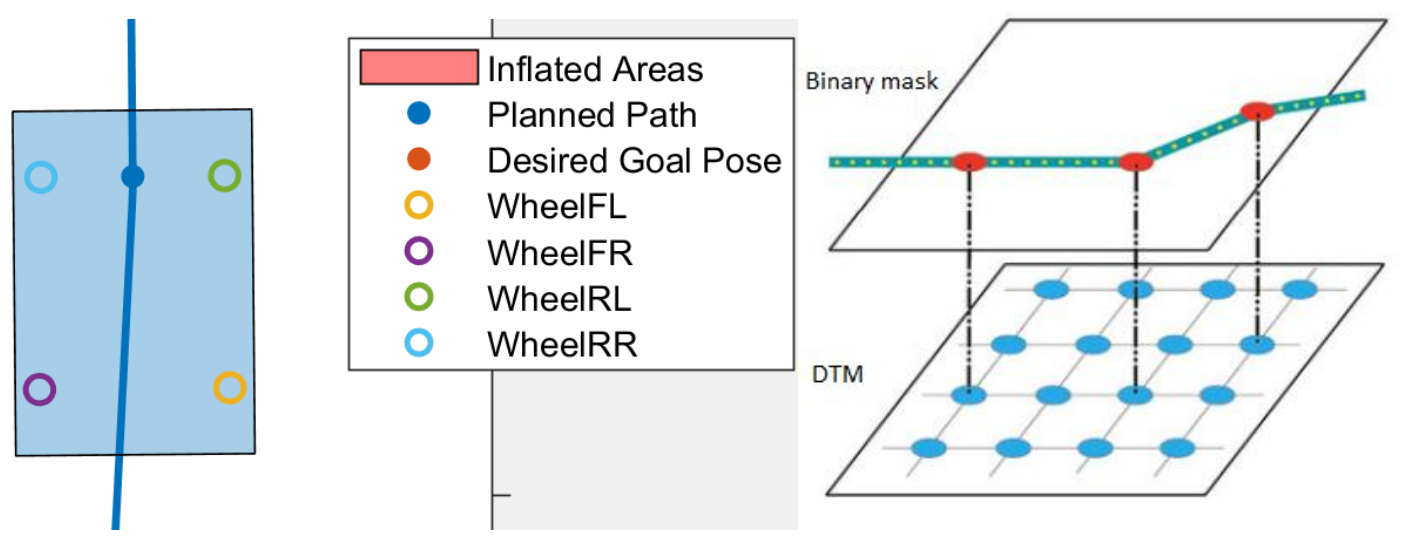

Fig. 3. (Left) Visual example of wheel contact points and (Right) its relation with the binary mask and the DTM. 


\section{Prototype testing and validation}

In the final phase of the project, given the period of restrictions due to the covid-19 pandemic, running the validation tests in a vineyard was impossible, therefore, the rover was tested in an airfield near the town of Rivoli (TO), in Piedmont. This site neither has vineyards nor a particularly rough terrain, unlike the typical operational environment. Since the conformation and the specificities of the vineyard had to be replicated, the DTM and the starting orthophoto of the airfield were modified to simulate the obstacles' presence. In addition to the previously described sensors, a Leica MS50 total station (TS) plus a Leica GS14 GNSS receiver were used to track a prism mounted on the same axis of the Piksi GNSS antenna, to provide the ground truth useful for validating the trajectory. The control inputs are generated by the Pixhawk board which use a navigation filter based on the Extended Kalman Filter.

\section{RESULTS}

Regarding the path planning algorithm results, several simulations were conducted to investigate the probability for the $\mathrm{RRT}^{*}$ to find a path in different scenarios with an increasing difficulty to complete the task. Changing the scenario greatly increase the time required by the code to provide a valid path, i.e. a path that falls within the imposed constraints. To do so, several tests were made running several cycles of the code in a fixed time frame (24 minutes), changing the number of iteration and constraints. The analysis lead to the conclusion that the parameters mostly affecting the search for a route are the distance between the poses and the inflation radius of the obstacles. The increase in the distance between one pose and the next brings some benefits since, by increasing the distance between the nodes, the ramifications generated by the algorithm can grow further on the map. The reduction of the inflation radius involves an enlargement of the Voronoi regions of the map, with the consequent increase of the probability of the algorithm to extend the ramifications also within rows. For simplicity, Fig. 4 shows the results obtained in a specific scenario with the following parameters:

- Pose distance: $0.2 \mathrm{~m}$ \& Obstacle inflaction radius: $0.475 \mathrm{~m}$ (orange line);

- Pose distance: $0.3 \mathrm{~m}$ \& Obstacle inflaction radius: $0.35 \mathrm{~m}$ (white line).

The second graph represents the average time it takes for the algorithm to find a path to validate. These simulations were also useful to identify an ideal number of initial maximum iterations (10000), since excellent success probability and computation time results were obtained with that number, in multiple scenarios. 

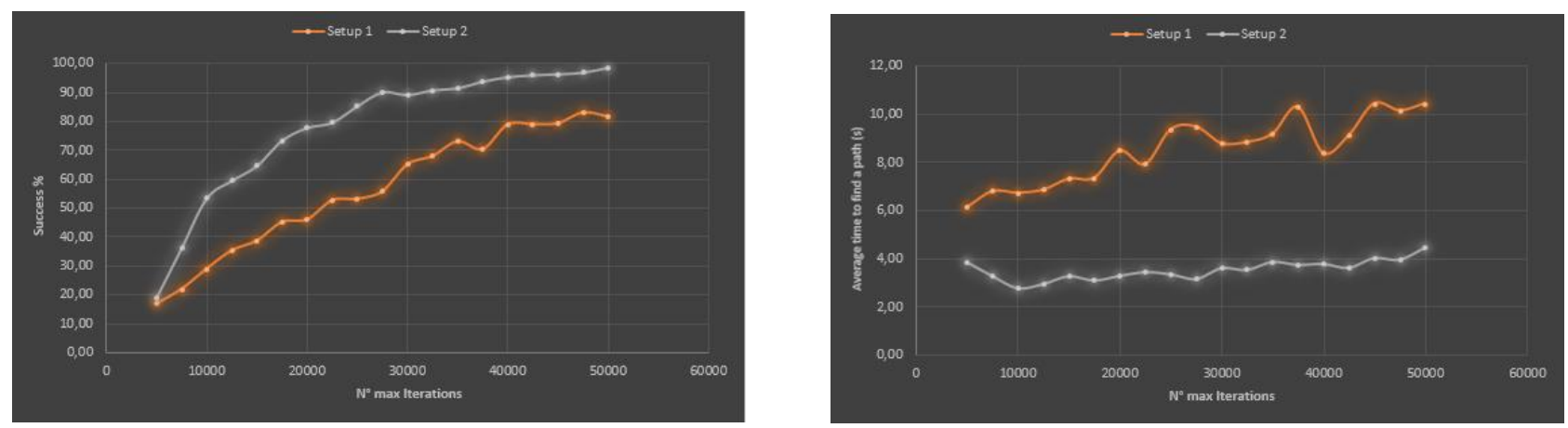

Fig. 4. (Left) Probability percentage of success to find a path with respect to the number of algorithm iteration. (Right) Average time to find a path with respect to the number of iteration.

Concerning the navigation results, the path planner's set of waypoints was compared with the navigation solution obtained by the autopilot navigation filter and with the real trajectory followed by the rover and measured by the TS (Fig. 5). The Root Mean Squared Error (RMSE) of the 2D solution and its standard deviation are reported in Table 1. It is possible to observe that the difference between the trajectories is minimal along straights and falls within the necessary values to be obtained for this type of application. It can be stated that this difference is mostly caused by a problem with wheel camber due to the fully discharged shock absorbers, which lead the vehicle to skid to the right during what should be a straight line. Something different happens if the behavior of the vehicle is observed in curves, where the difference between trajectories is relevant. The cause of this behavior during curves is due to the vehicle speed that we were forced to set that results unsuitable for precision maneuvers. Since at low speeds $(1 \mathrm{~m} / \mathrm{s})$ the vehicle does not navigate fluidly we were forced to set a speed value of $1.5 \mathrm{~m} / \mathrm{s}$ to ensure smooth driving. These errors are further accentuated if we consider the inherent problems due to the fully discharged shock absorbers above mentioned.
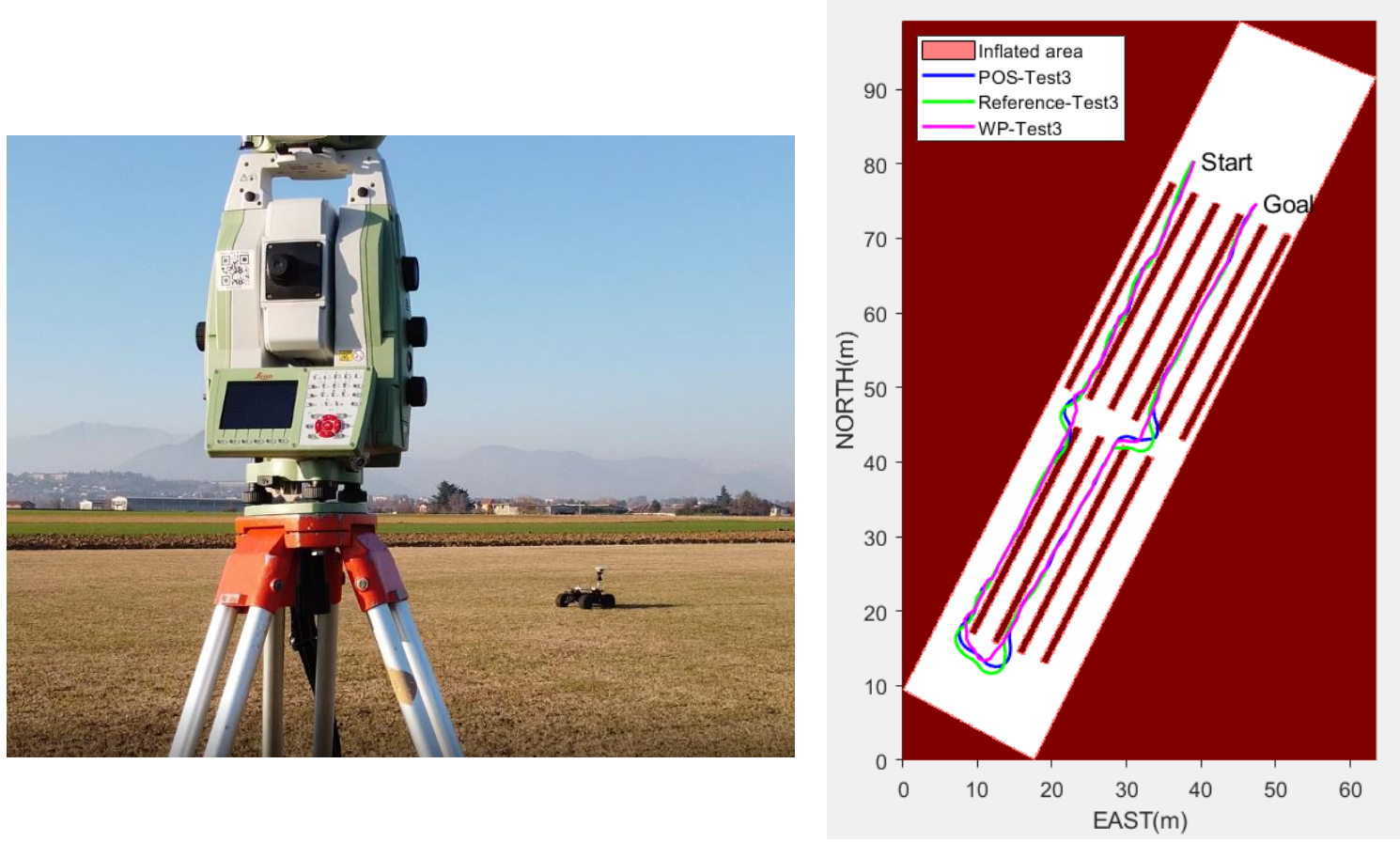

Fig. 5. T(Left) Total Station tracking the vehicle. (Right) TS, EKF and Waypoints trajectories plotted onto the binary mask with the presence of virtual rows. 
Table 1. Planar navigation error of the real trajectory with respect to the planned one obtained by the algorithm and the estimated one obtained by the autopilot.

\begin{tabular}{lll}
\hline Comparison & 2D RMSE & Std. Dev. \\
\hline Real(TS) vs Autopilot & $0.294 \mathrm{~m}$ & $0.301 \mathrm{~m}$ \\
\hline Real (TS) vs Path Plan & $0.384 \mathrm{~m}$ & $0.451 \mathrm{~m}$ \\
\hline
\end{tabular}

\section{CONCLUSIONS}

This work aims to realize a vehicle that can navigate autonomously in uneven terrain independently through GNSS positioning and a path planner algorithm. The main contribution to achieve this goal was the realization of an algorithm able to generate a safe and efficient route with respect to different parameters such as distance, slope and its variation, guarantying precision navigation route through very hard environments, like a vineyard. The $\mathrm{RRT}^{\star}$ planning algorithm that was used and the superstructure that was created all around it, to adapt the algorithm to the purpose and the problems of this type of terrain, allows to accomplish the task, thanks to the collaboration of a UAV that, through an air-photogrammetric survey, provide to the algorithm high-resolution maps with centimeterlevel accuracy. The assembly and selection of sensors made it possible to instrument a commercial rover and test it in a real case study. Navigation was entrusted to the Pixhawk autopilot, which performed the planned mission through the proposed algorithm. The accuracy of the navigation solution was calculated by comparing it with the ground truth. The results obtained show that the planner can find a path in a complex vineyard environment with very few fails and the UGV follow the route respecting the accuracy required in these kinds of applications.

\section{REFERENCES}

Wang, H., \& Noguchi, N. (2019). Navigation of a robot tractor using the centimeter-level augmentation information via Quasi-Zenith Satellite System. Engineering in Agriculture, Environment and Food, 12(4), 414-419.

Emmi, L., Le Flécher, E., Cadenat, V., \& Devy, M. A hybrid representation of the environment to improve autonomous navigation of mobile robots in agriculture. Precision Agriculture, 1-26.

Zhang, Z., Li, P., Zhao, S., Lv, Z., Du, F., \& An, Y. (2021). An Adaptive Vision Navigation Algorithm in Agricultural loT System for Smart Agricultural Robots. CMC-COMPUTERS MATERIALS \& CONTINUA, 66(1), 1043-1056.

Michael W Otte. A survey of machine learning approaches to robotic path-planning. University of Colorado at Boulder, Boulder, 2015.

Karaman, S., \& Frazzoli, E. (2011). Sampling-based algorithms for optimal motion planning. The international journal of robotics research, 30(7), 846-894.

Zoto, J., Musci, M. A., Khaliq, A., Chiaberge, M., \& Aicardi, I. (2019, June). Automatic path 
planning for unmanned ground vehicle using uav imagery. In International Conference on Robotics in Alpe-Adria Danube Region (pp. 223-230). Springer, Cham. 